\title{
Papers
}

\section{Timing of birth and infant and early neonatal mortality in Sweden 1973-95: longitudinal birth register study}

\author{
Z C Luo, J Karlberg
}

\begin{abstract}
Objective To assess the impact of time of birth on infant mortality and early neonatal mortality in full term and preterm births.

Design Analysis of data from the Swedish birth register, 1973-95.

Participants 2102324 spontaneous live births of infants without congenital malformation.

Outcome measurements Absolute and relative risk of infant mortality, early neonatal mortality, and early neonatal mortality related to asphyxia.

Results Infant mortality, early neonatal mortality, and early neonatal mortality related to asphyxia were higher in infants who were born during the night (9 pm to $9 \mathrm{am}$ ) compared with those born during the day for 1973-9, 1980-9, and 1990-5. The difference was more dramatic for preterm infants. The largest difference was observed during 1990-5, when there was a $30 \%$ increase in early neonatal mortality (relative risk $1.31,95 \%$ confidence interval 1.10 to $1.57)$ and a $70 \%$ increase in early neonatal mortality related to asphyxia $(1.70,1.22$ to 2.38$)$ in preterm infants born during the night compared with rates for preterm infants born during the day. A detailed analysis over 24 hours revealed two "high risk" periods: between $5 \mathrm{pm}$ and $1 \mathrm{am}$ and around $9 \mathrm{am}$. Conclusions Infants born during the night have a greater risk of infant and early neonatal mortality and early neonatal mortality related to asphyxia than those born during the day. There has been no improvement over the past two decades. The problem is more serious for preterm births and was even worse in the 1990s. Shift changes and the hours immediately after such changes are high risk periods for neonatal care.
\end{abstract}

\section{Introduction}

In recent years several studies have reported a higher early neonatal mortality, particularly mortality related to asphyxia, in infants born during the night than in those born in the day. ${ }^{1-3}$ This has important implications for health care as millions of births take place at night throughout the world. An exploration of the time of birth in relation to such mortality will help us to assess the importance of this problem in preterm infants-those most at risk. We assessed whether infant mortality and early neonatal mortality is related to the time of birth in both full term and preterm infants born in Sweden.

\section{Methods}

We used data from the Swedish birth register, 1973-95..$^{4-6}$ The registry came into effect in 1973 and covers virtually all births in the country. A wide range of data about mothers and newborns are collated from interviews with mothers and from various records. The data include records on the exact time of birth. The inputs include standardised sets of forms used in all antenatal clinics and delivery units and during paediatric examinations of newborn infants. The records are linked to the registry of population that includes the death registry. ${ }^{5}$ Data on mortality during the first year of life are merged with the data from the birth registry. There were 2392263 valid births recorded by the registry during 1973-95. To assess the effect of the time of birth in comparison with the recent result by Heller ${ }^{1}$ we retained only those data on spontaneous live births (and thus not planned time of birth) of infants without considerable congenital malformation (2 102324 infants). We also assessed which hours of birth over 24 hours were "high risk" periods for infant and early neonatal mortality and early neonatal mortality related to asphyxia.

During the study period there were no alterations in routines surrounding staff shift changes in Sweden. The obstetric nurses change shifts at 7 am, and the nurses have full responsibilities for each delivery. The obstetricians and neonatalogists change shifts at $8 \mathrm{am}$. Larger hospitals have a neonatalogist on call 24 hours; medium and small hospitals have a senior paediatrician on call 24 hours. The general principle is that all complicated deliveries will have a neonatalogist present, and premature and sick babies will be cared for in special neonatal wards. Early neonatal mortality was similar in babies born in large cities and those born elsewhere (1.57 v 1.53 per $1000, \mathrm{P}=0.51)$, an indication of homogeneity of neonatal care across the country.

Night time births refers to births that occurred in the hours from $9 \mathrm{pm}$ to $7 \mathrm{am} .{ }^{1}$ Early neonatal death refers to death in the first six days of life. ${ }^{17}$ The occurrence of death, as noted from the Swedish death register, was cross validated by the hospital based Swedish birth register. In a few cases of discrepancy between the hospital based records and the death register, we used

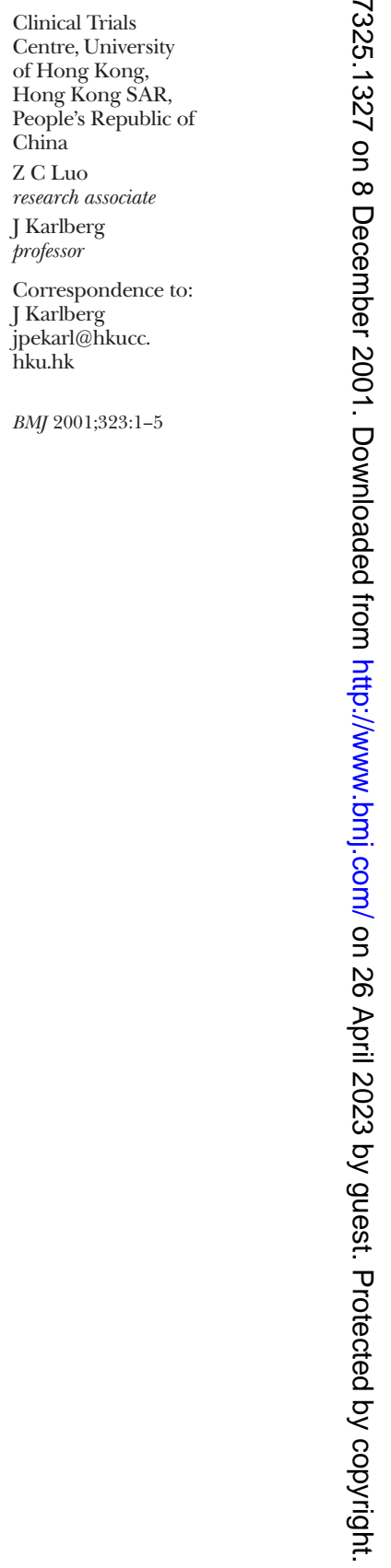


Table 1 Births and infant mortality statistics for live births in Sweden, 1973-95

\begin{tabular}{lccc} 
& All births 1973-95 & $\begin{array}{c}\text { All births excluding caesarean } \\
\text { deliveries }\end{array}$ & $\begin{array}{c}\text { All births excluding caesarean deliveries and infants } \\
\text { with severe congenital malformation }\end{array}$ \\
\hline No of births & 2392 263 & 2134309 & 2102324 \\
\hline No of deaths in first year & 15534 & 10732 & 7809 \\
\hline Infant mortality (per 1000 live births) & 6.5 & 5.0 & 3.7 \\
\hline
\end{tabular}

Table 2 Infant mortality and mortality related to asphyxia (number of deaths per 1000 live births) during first year of life for spontaneous live births of infants without congenital malformation according to time of birth, Sweden 1973-95

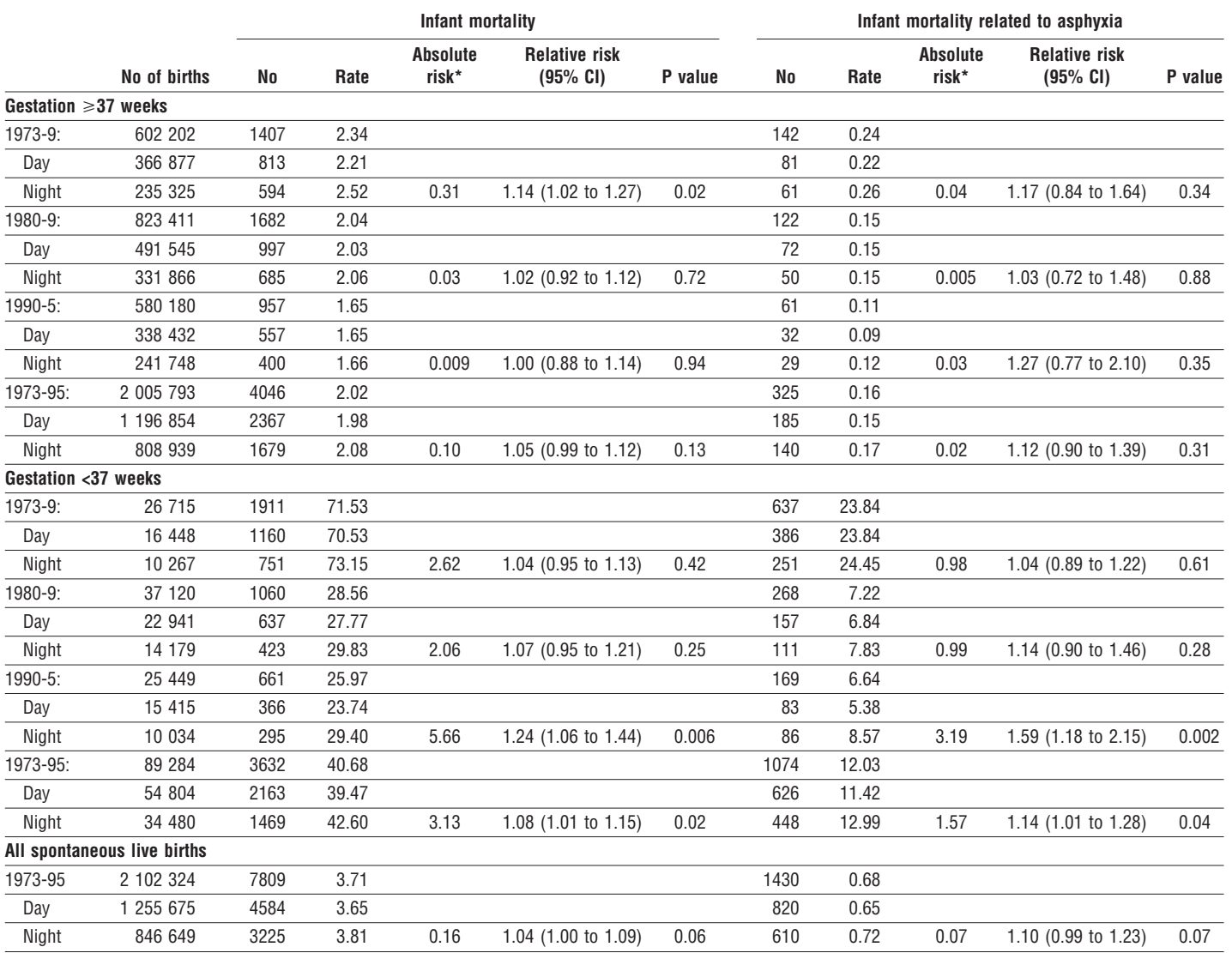

*No of deaths per 1000 births.

the hospital records. We recorded the cause of death as defined by ICD-8 (international classification of diseases and related health problems, eighth revision) for 1973-86 and ICD-9 (ninth revision) for 1987-95. ${ }^{4}$ Asphyxia related deaths were represented by code ICD-8 code 776 and ICD-9 codes 767, 768, 769, and 770.

We computed the absolute and relative risks to evaluate the impact of the time of birth on the risk of infant and early neonatal mortality and early neonatal mortality related to asphyxia. We assessed preterm and term births separately in the analysis of infant and early neonatal mortality. The analyses were done for 1973-9, 1980-9, and 1990-5 separately so that we could make comparisons with results from other countries for $1990-5^{178}$ and assess changes over decades.

\section{Results}

There were around 2.39 million live births during 1973-95. Table 1 shows the average infant mortality per thousand births. Early neonatal deaths (in the first six days) accounted for more than half of all deaths during the first year of life. A steady decline in infant mortality and early neonatal mortality was observed from 1973 to 1995 (fig 1). There was a slight increase in early neonatal mortality related to asphyxia during

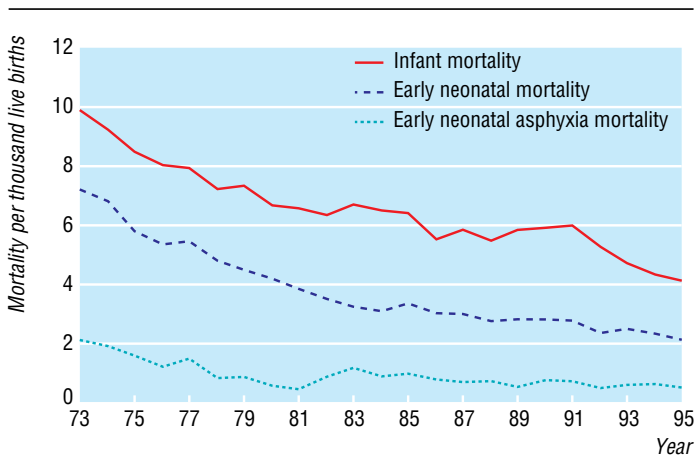

Fig 1 Infant mortality for spontaneous live births of infants without congenital malformation, Sweden 1973-95 (Cochran-Armitage test for trend, $P<0.0001$ for different rates during 1973-95) 
1982-3, but otherwise there was a general downward trend from 1973 to 1995.

There was a higher risk of infant mortality and mortality related to asphyxia during the first year of life in infants born during the night than in those born during the day (table 2). The difference was mostly small but was greater for preterm infants. The most striking difference was in 1990-5, with an increase of about $60 \%$ in the risk of mortality related to asphyxia in preterm infants born during the night compared with preterm infants born during the day. The results for early neonatal mortality - that is, in the first six days-were similar to those for mortality in the first year (table 3). The increase of such mortality in 1990-5 was about $30 \%$ for early neonatal mortality (relative risk $1.31,95 \%$ confidence interval 1.10 to 1.57 ) and about $70 \%$ for early neonatal mortality related to asphyxia $(1.70,1.22$ to 2.38$)$ in preterm infants born at night.

Figure 2 shows the changes in mortality over 24 hours in the time of birth in one and two hour intervals. A high risk period occurred between 5 pm and $1 \mathrm{am}$, with another short high risk period around $9 \mathrm{am}$. There was a low risk period between 2 am and $4 \mathrm{pm}$, except for the short peak around $9 \mathrm{am}$. The largest difference was in early neonatal mortality related to asphyxia: infants born during the hours of highest risk (around $9 \mathrm{am}$ and $9 \mathrm{pm}$ ) had almost double the risk of such mortality compared with infants born during the hours of lowest risk (around 4 am and 3 pm).

The changes in mortality followed similar trends over 24 hours during weekdays (Monday-Friday) and at the weekend (Saturday and Sunday), as shown for early neonatal mortality in figure 3. Early neonatal mortality was higher from 5 pm to 1 am and around 9 am for both weekdays and weekend days. The absolute early neonatal mortality decreased dramatically over the three periods, though the fluctuations over the 24 hours followed a similar trend with "high risk" periods around 9 am and 9 pm to 1 am (fig 4).

\section{Discussion}

Our observations confirm the previous reports from Germany and the United Kingdom that infants born at night have a higher risk of early neonatal mortality and mortality related to asphyxia than infants born during the day. ${ }^{1-3}$ The new message from our observation is that this problem is much more serious for preterm infants and that shift changes and the hours immediately after are high risk periods. Furthermore, there was no improvement in the quality of neonatal health care in the night compared with that in the day over the decades we studied. There was an even greater relative risk of early neonatal mortality and mortality related to asphyxia for infants born at night during the 1990s.

Strengths and weaknesses in relation to other studies Quality of data is an important consideration for studies on neonatal mortality. There is a large difference in early neonatal mortality reported within developed countries. ${ }^{178}$ This may be due to true differences among populations but could also be due to a lack of consistency in collecting, recording, and coding data on births and deaths in different countries. For 1991-5
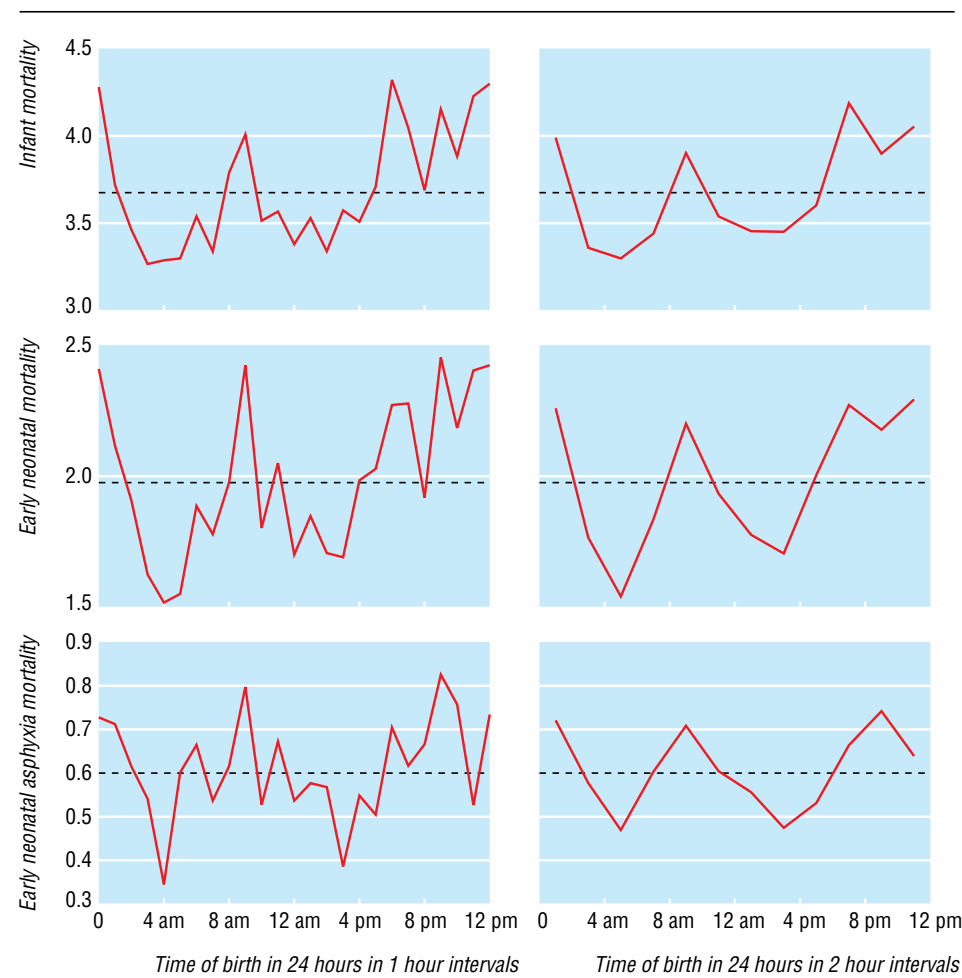

Fig 2 Changes in mortality over 24 hours according to time of birth by one or two hour intervals for spontaneous live births of infants without congenital malformation, Sweden 1973-95. Dashed lines represent reference mean values over 24 hours $\left(\chi^{2}\right.$ test for difference over 24 hours, $P<0.0001$ for infant mortality and early neonatal mortality by both one and two hour intervals; $\mathrm{P}=0.005$ and 0.007 for early neonatal mortality related to asphyxia by one and two hour intervals, respectively)

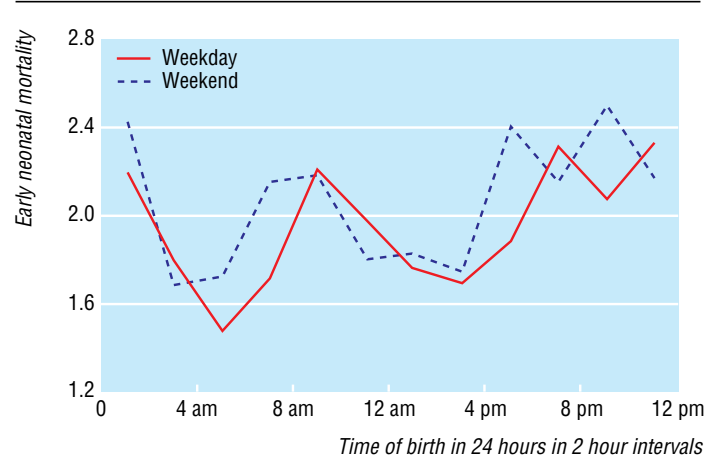

Fig 3 Changes in early neonatal mortality over 24 hours according to time of birth by two hour intervals and day of week for spontaneous live births of infants without congenital malformation in Sweden $\left(\chi^{2}\right.$ test for difference over 24 hours: $P<0.0001$ for weekdays, $\mathrm{P}=0.036$ for weekend)

the observed early neonatal mortality for live full term infants without congenital malformation was 0.15 per thousand live births in Germany ${ }^{1}$ and 0.32 in Sweden. In Canada the rate was 0.39 for $1992-4,{ }^{5}$ and in the United States it was 0.27 for $1995 .^{7}$ Our results are similar to those from studies in Canada and the United States, while the German study gave an exceptional low value and may be less accurate. The observed difference in early neonatal mortality between infants born during the day and night was much greater in the German study than that observed in our study. 
Table 3 Early neonatal mortality and mortality related to asphyxia (number of deaths per 1000 live births) during first six days for spontaneous live births of infants without congenital malformation according to time of birth, Sweden, 1973-95, compared with data from Germany, 1990-5

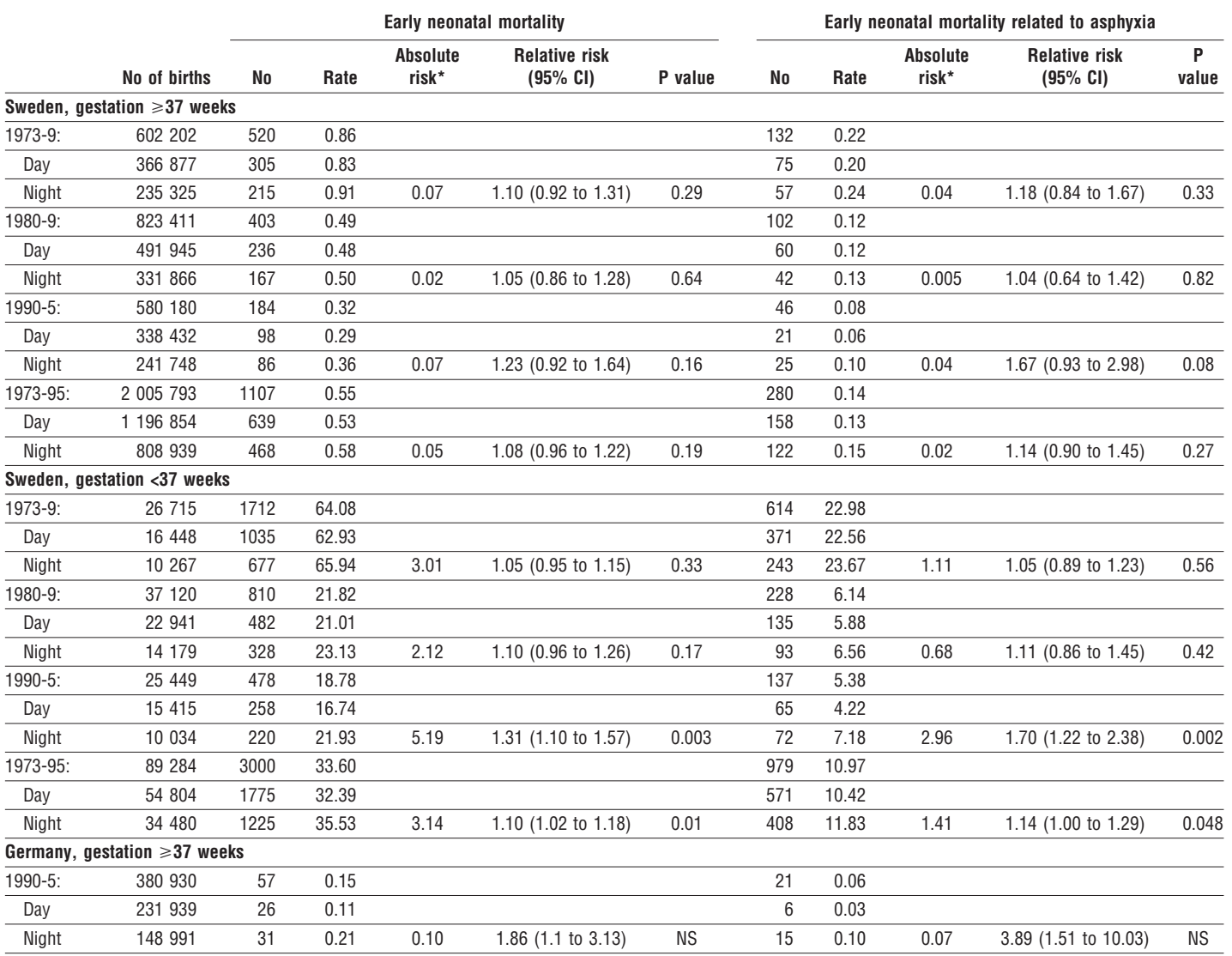

*No of deaths per 1000 births.

The data from the Swedish birth registry are of high quality. ${ }^{4}$ As virtually all births were recorded there is no problem with sample error, therefore all parameters estimated here represent the true value of the population. There is no need to estimate confidence intervals, although we have given confidence intervals for the relative risks for illustration.

Sweden is a typical developed country with good social welfare and health care. The observed results would probably apply elsewhere, such as in Canada,

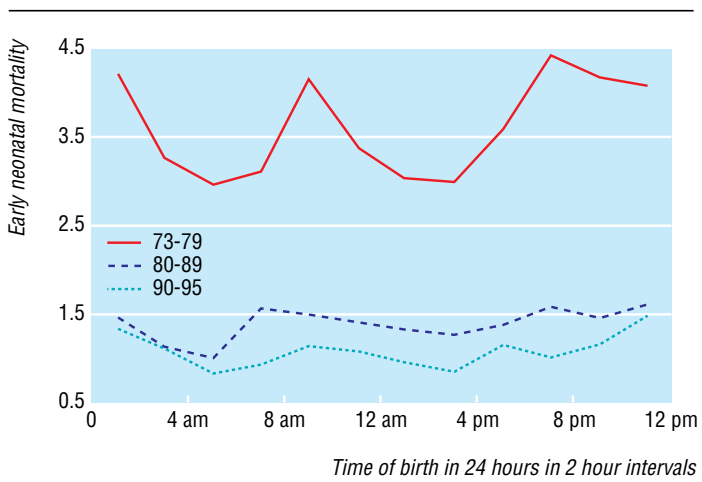

Fig 4 Changes in early neonatal mortality over 24 hours according to time of birth by two hour intervals for spontaneous live births of infants without congenital malformation in Sweden, 1973-9, 1980-9, and 1990-5 ( $\chi^{2}$ test for difference over 24 hours: $P<0.0001$ for $1973-9, P=0.08$ for $1980-9, P=0.005$ for $1990-5$ )
Japan, Singapore, Hong Kong, and European countries, but may not be applicable to developing or underdeveloped regions or countries without such social welfare and healthcare systems. However, we would expect to see more dramatic differences in infant and early neonatal mortality between infants born during the night and day in less developed regions or in countries with a less effective healthcare system.

Possible mechanism and implications

Even with the improvements in health care the greatest difference between mortality in infants born day or night was during 1990-5. There was about 30\% higher early neonatal mortality and $70 \%$ higher neonatal mortality related to asphyxia in preterm infants born at night compared with preterm infants born during the day. There was also a large increase in absolute and relative risk of early neonatal mortality for preterm infants born at night in the 1990s compared with the 1980 s. To some extent, there was no improvement or even a worsening in the quality of night time neonatal care compared with the daytime neonatal care in the 1990s. This certainly raises our concern, and efforts should be made to eliminate this difference. The benefit would be enormous considering the millions of births worldwide. The underlying causes are not clear and may be due to excess workloads, inadequate or less experienced staff on night shifts, or out of date systems for managing shift changes within hospitals. 


\section{What is already known on this topic}

Infants born at night have a greater risk of early neonatal mortality and early neonatal mortality related to asphyxia than those born during the day

The causes are unclear but may be related to insufficient or less experienced staff or excess workload during the night

\section{What this study adds}

In Sweden the relative risks of infant and early neonatal mortality and mortality related to asphyxia for infants born during the night compared with during the day did not diminish during 1973-95, are greater for preterm infants, and were greater in the $1990 \mathrm{~s}$

There are two "high risk" periods for early neonatal mortality: 5 pm to 1 am and around 9 am

The exact reasons are unclear but better vigilance and an improvement in shift changes may be required to improve neonatal health care further

A more in depth analysis over 24 hours showed that there were actually two high risk periods for neonatal care: $5 \mathrm{pm}$ to $1 \mathrm{am}$ and around $9 \mathrm{am}$. The first risk period started from the end of the normal day shift and extended to the midnight shift. The second one, around $9 \mathrm{am}$, was immediately after day shift staff were taking over responsibilities. This pattern indicates that shift changes and the periods immediately after are high risk periods for neonatal care. Not enough vigilance when staff have just started their shift may be a problem, and neonatal healthcare staff should definitely be more watchful during shift changes and the periods immediately after. Another problem may be due to insufficient time available for close observation during the shift changes. The accumulation of difficult cases during the night may also explain the higher risk of early neonatal deaths around $9 \mathrm{am}$. The exact answers to the mechanisms and causes should be identified by further investigations.

We thank all hospital staff for their work in collecting and filing data for the Swedish birth register, and the Children's Hospital of the University of Goteborg for providing access to the data.

Contributors: JK was responsible for the study design and theoretical framework and participated in data analysis, interpretation of results, and manuscript writing. ZCL participated in the study design, interpretation of results, and manuscript writing and was responsible for the data analysis. JK is guarantor.

Funding: Clinical Trials Centre and the Department of Pediatrics, University of Hong Kong.

Competing interest: None declared.

1 Heller G, Misselwitz B, Schmidt S. Early neonatal mortality, asphyxia related deaths, and timing of low risk births in Hesse, Germany, 1990-8: observational study. BMJ 2000;321:274-5.

2 Stewart JH, Andrews J, Cartlidge PHT. Numbers of deaths related to intrapartum asphyxia and timing of birth in all Wales perinatal survey, 1993-95. BMJ 1998; 316:657-60.

3 Chalmers JWT, Shanks E, Paterson S, McInneny K, Baird D, Penney G. Scottish data on intrapartum related deaths are in the same direction as Welsh data. BMJ 1998;317:539.

4 Cnattingius S, Ericson A, Gunnarskog J, Kallen B. A quality study of a medical birth registry. Scan J Soc Med 1990;18:143-8.

5 Cheung YB, Yip PS, Karlberg J. Mortality of twins and singletons: a varying-coefficient approach. Am J Epidemiol 2000;152:1107-16.

6 Cheung YB, Yip PSF, Karlberg J. Parametric modeling of neonatal mortality in relation to size at birth. Stat Med 2001;20:2455-66.

7 Kramer MS, Demissie K, Yang H, Platt RW, Sauve R, Liston R. The contribution of mild and moderate preterm birth to infant mortality. JAMA 2000;284:843-9.

8 Pan American Health Organization. II. Health by population group. In Health in the Americas. Washington, DC: PAHO Scientific Publication, 1998

(Accepted 29 August 2001) 